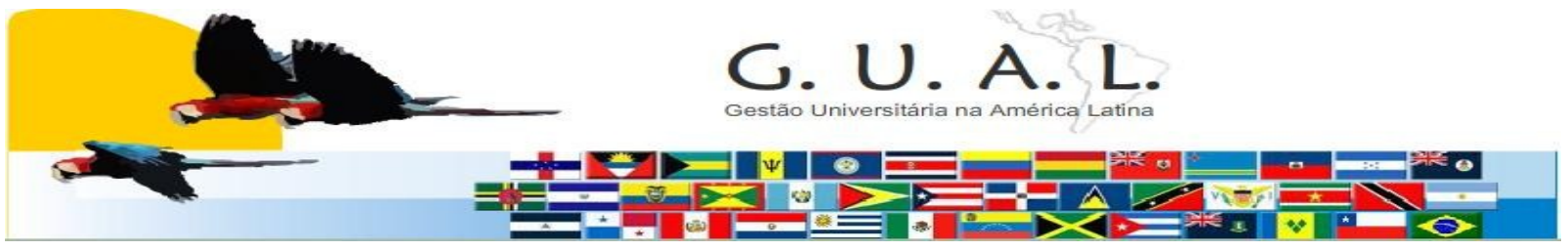

ISSN 1983-4535

\title{
LA GESTIÓN DEL CONOCIMIENTO EN Y PARA LA UNIVERSIDAD
}

Roberto Ismael Vega, UNMDP

\section{INTRODUCCIÓN}

El presente trabajo parte de la hipótesis que la Universidad Latinoamericana ha avanzado en el desarrollo y la aplicación de los conocimientos científicos de los últimos dos siglos, incorporándolos a las diferentes currículas de las carreras que ofrece. Esto es particularmente válido para las disciplinas que sirven a la gestión de las organizaciones, entendiendo por tal desde la fijación de políticas hasta la administración de los medios para implementarlas.

No obstante no se verifica que este conocimiento haya sido aplicado en su propia gestión, que sigue moviéndose con gran improvisación y donde no parece que el conocimiento tenga un lugar destacado.

Esta carencia conlleva, en una época de grandes mutaciones, a la imposibilidad de contar con estructuras flexibles y cuerpos de gestores calificados que permitan que la Universidad se mantenga acorde a estos cambios y constituya un motor para el desarrollo de nuestras sociedades.

\section{ANTECEDENTES HISTÓRICOS}

La revolución industrial puso el conocimiento como insumo estratégico de todos los procesos productivos y también en la base de las relaciones sociales vigentes, produciendo el encumbramiento definitivo de la burguesía en desmedro de las estructuras de casta vigentes hasta esa época.

La Universidad respondió a esta situación con dos modelos. Por un lado la creación de la Universidad Napoleónica (1808) que constituyó un intento de dotar a Francia de los 
profesionales que ésta necesitaba para recuperar el terreno perdido frente a Inglaterra en el proceso de industrialización y transformación de sus estructuras socioproductivas. Este modelo se basó en Escuelas (Puentes y Caminos, Administración, etc) que dan origen al sistema de Facultades que luego importaría América Latina, con la salvedad de que en su origen el conocimiento es orientado a la solución de problemas específicos de la Nación y en su traslación hacia nuestro medio esta impronta se perdió.

Por otra parte en Alemania Von Humboldt reforma la Universidad de Berlín (1802), centrándola en la creación de nuevos conocimientos. Esto dio origen a un espectacular desarrollo de la ciencia en la Alemania del siglo XIX, el cual se tradujo en que son de este origen la inmensa mayoría de los adelantos científicos - tanto en ciencias naturales como sociales- que se verifican en dicho siglo.

Con posterioridad y ya avanzado el siglo XIX, dos países de muy diferente historia y cultura también asumen la Universidad como palanca de desarrollo. Japón, con la instauración de la dinastía Meiji (1867), crea la Universidad Imperial de Tokio y en ella procura desarrollar toda la ciencia occidental y sus aplicaciones, para ello incorpora profesores de las universidades europeas y posteriormente envía a sus graduados a perfeccionarse en las mismas. Inicia de este modo un proceso de modernización e industrialización que convertiría a Japón en potencia industrial en los albores del siglo XX. Por otra parte Estados Unidos de Norteamérica sanciona en 1862 la Land Grant Act que dispone la donación de tierras federales a los nuevos Estados para que con su usufructo se creen universidades destinadas al desarrollo de las artes mecánicas y agrícolas, que constituyen la base del ulterior desarrollo industrial de este país.

Finalmente ya en pleno siglo XX son notorias las experiencias de la ex URSS y de Cuba, que mediante el impulso decidido dado a su sistema universitario lograron en poco tiempo reducir sustancialmente la brecha tecnológica que los separaba del mundo desarrollado y convertirse la primera, en una potencia industrial y tecnológica y la segunda, con medios mucho más modestos, en un centro de excelencia en ciencias médicas y biotecnología.

Mientras tanto en Latinoamérica las primeras universidades creadas por los españoles reprodujeron su carácter eclesial y retórico, convirtiéndose en "universidades de abogados", destinados a la conducción política de las nuevas naciones y a la protección de la naciente oligarquía terrateniente y rentística. 
Es así que en 1959, al asumir Risieri Frondizi ${ }^{1}$ el rectorado de la Universidad de Buenos Aires, ésta contaba con nueve dedicaciones exclusivas y la investigación prácticamente estaba exclaustrada.

Esta situación si bien ha mejorado sensiblemente aún mantiene ciertas características que podemos resumir en lo siguiente:

- Investigación anárquica. Prácticamente no han existido en forma explícita políticas de investigación que tiendan a sinergizar los proyectos que se desarrollan, observándose una muy escasa articulación entre grupos que pretenden trabajar sobre temáticas semejantes. Esta situación se refuerza con una fuerte tendencia a investigaciones monodisciplinares que por su mismo carácter, dejan fuera de su incumbencia vastos campos problemáticos de nuestra sociedad.

- Dependencia externa en ciencias biológicas y exactas. Dado que tradicionalmente nuestros investigadores han recibido su formación en centros extranjeros (de Europa y EEUU), sus proyectos de investigación permanecen vinculados a los de los grupos en los cuales han realizado sus doctorados y posdoctorados, ya que ese es el modo de poder seguir vinculados a centros de excelencia y lograr que sus trabajos sean publicados en revistas indexadas de prestigio internacional.

- Escasez de fondos destinados a Investigación: En nuestro país se destina sólo el $0,4 \%{ }^{2}$ del PBI a la producción científica y en su casi totalidad se trata de fondos públicos, ya que las empresas rara vez están dispuestas a financiar proyectos que impliquen riesgos y han preferido históricamente, la compra acrítica de tecnología desarrollada en otros países.

Para cerrar este punto resulta ilustrativo observar como la Provincia de Québec, en Canadá, asumió su "subdesarrollo relativo" mediante la creación de la Universidad de Québec (1972), constituida mediante una red que incluye un Instituto de Investigaciones Biológicas, una Teleuniversité para cursos a distancia y cinco sedes universitarias ubicadas en diferentes regiones de la provincia, y que han tenido como objetivo fundamental impulsar el desarrollo socioeconómico del interior quebequois.

Es importante señalar en este punto que todo lo anteriormente consignado requirió un importante esfuerzo al interior de los respectivos sistemas universitarios y de cada una de sus universidades para lograr niveles de racionalidad, eficacia y eficiencia, así como una consistente coordinación sistémica para hacer efectivo el esfuerzo y lograr el impacto que se perseguía.

\footnotetext{
${ }^{1}$ Frondizi Risieri. "La Universidad en un Mundo de Tensiones". 1971 Edit. Paidos Bs. Aires

${ }^{2}$ Como dato ilustrativo Brasil invierte el 1\% de su PBI en Desarrollo científico.
} 


\section{TENDENCIA ACTUAL}

Afortunadamente se observa actualmente en el ámbito de nuestros sistemas universitarios un mayor énfasis en promocionar la investigación y la transferencia tecnológica al medio socioproductivo. Esto se verifica en nuestro país a través de los siguientes hechos que se han venido constatando en los últimos años:

- Mayor presupuesto asignado al sistema universitario. En los últimos cinco años se verifica un aumento anual a valores constantes $^{3}$ de un $9 \%$ promedio, lo cual significa un importante aporte real, ya que el aumento de la matrícula no registra estos índices.

- Régimen de Incentivos a Investigadores: la sanción del Decreto 2427/03 de creación del régimen de Incentivos a investigadores, con un sistemas de categorías basado en la producción individual de cada uno ${ }^{4}$, ha representado un importante aliciente para el desarrollo de la investigación y sobre todo, para la aparición de nuevos grupos que encaran áreas hasta ahora inexploradas. Por otra parte este mecanismo ha forzado a que las propias universidades deriven mayor esfuerzo hacia la investigación presionadas por el creciente número de investigadores que requieren mayores y mejores equipamientos e instalaciones.

- Intentos de políticas de acercamiento y colaboración entre universidades e institutos del Estado dedicados a la investigación o al fomento de la misma (Agencia de Desarrollo Científico y Tecnológico, Conicet, Cic. INTA, Conea, Inti, etc.). Finalmente en este sentido ha sido de trascendencia, al menos en cuanto demostración del interés del Estado en el tema, la creación del Ministerio de Ciencia y Tecnología.

No obstante la transferencia al medio socioproductivo aún se mantiene en un grado incipiente de desarrollo, si se la compara con los niveles alcanzados en otros países. Al respecto creemos que existen algunos motivos para esta situación que son inherentes a esta problemática en todo el mundo ${ }^{5}$ y otros que son más propios de nuestra idiosincrasia. Una ligera revista de los mismos es la siguiente:

- Problemas culturales. En general nuestra sociedad y particularmente nuestra clase dirigente no percibe a la Universidad como posible fuente de asesoramiento para el tratamiento de problemas de base tecnológica y/o científica. Por otra parte las

\footnotetext{
${ }^{3}$ Cálculo de actualización sobre la base de índices de precios minoristas INDEC 2003 al 2007

${ }^{4}$ Ver Arana, Marta "El programa de incentivos bajo la Lupa. El caso de la UNMDP” 2005 Edit. Balder y García de Fanelli, Ana María “Universidad, Organización e Incentivos” 2005. Edit. Miño y Dávila.

${ }^{5}$ Ver Fanfani, Emilio (Compilador) "Universidad y Empresa” 1993. Miño y Dávila Editores. Y Sutz, Judith

"Universidad y Sectores Productivos" 1994. Edit. Centro Editor de Am
} 
racionalidades imperantes en la empresa, con su particular valoración del tiempo y la urgencia, no resultan compatibles con el modelo universitario de gestión, burocrático y pesado, ni con los tiempos y objetivos de la investigación como actividad, que siempre sacrificará tiempos en aras de la búsqueda de la verdad científica.

- Prejuicios internos. Nuestra Universidad Latinoamericana está ideológicamente imbuida de un ideario según el cual no resulta éticamente aceptable involucrar esfuerzos de una organización costeada por el pueblo, para resolver problemas de organizaciones lucrativas. Para salvar este escollo será menester demostrar cabalmente el beneficio recíproco que puede emerger de la colaboración Universidad - Empresa y, fundamentalmente y acorde a lo que las ideas schumpeterianas ${ }^{6}$ pusieron de manifiesto, las ventajas que derivarán para la sociedad como un todo de esta colaboración, a través de la innovación y la eficiencia de su aparato productivo.

- Pérdida de autonomía decisoria. Resulta evidente que las organizaciones del medio productivo sólo están dispuestas a financiar aquellas investigaciones y aplicaciones tecnológicas que pueden resultar rentables. En consecuencia es también evidente el riesgo que se corre si nuestras universidades empiezan a depender masivamente del aporte empresarial para desarrollar sus actividades de investigación, ya que la decisión acerca de que áreas y temas resultan prioritarios no dependerá de la racionalidad universitaria sino de las empresas dispuestas a pagar por ello. Este problema es hoy objeto de debate en prácticamente todo el campo universitario.

- Inequidad en la distribución. Por último no puede ignorarse que no todos los sectores de la Universidad están igualmente dotados de conocimientos y competencias que puedan ser objeto de transferencia al medio socioproductivo. En consecuencia a poco que una Universidad inicia sus actividades de transferencia se produce un conflicto por la distribución de los fondos que ésta genera entre aquellos directamente involucrados en su gestación, que pretenden ser especialmente beneficiados, y el resto de la comunidad universitaria que ve con recelo la aparición de un segmento de docentes que, por una diferente aplicación de su tiempo laboral, percibe mayores remuneraciones.

Para completar este panorama creemos importante destacar un par de cuestiones que constituyen en si mismas demandas que el Sistema Universitario deberá atender prioritariamente para lograr hacer productivo el conocimiento a todo nivel.

En primer lugar debe destacarse la necesidad de fomentar el trabajo interdisciplinario, ya que la realidad rara vez cabe en un área disciplinar, y resulta

\footnotetext{
${ }^{6}$ Schumpeter, Joseph. 1997, Fondo de Cultura Económica. Sostiene que sólo la innovación puede procurar calidad de vida a una nación al permitir a sus empresarios disfrutar durante un tiempo de la condición de productores monopólicos en función de la innovación tecnológica.
}

Rev. GUAL., Florianópolis, v.2, n. 1, p.47-61, ano 2009. 
evidente que si la Universidad pretende como tal asumir un rol protagónico en el desarrollo de sus comunidades de asiento, deberá previamente estar capacitada para emprender esta modalidad de trabajo.

Al respecto nuestras universidades no están particularmente dotadas. En primer término sus estructuras, mayoritariamente asentadas en el concepto de "Facultades", agrupan el conocimiento en compartimientos estancos razonablemente aptos para la formación de determinado perfil profesional, pero inconsistentes en cuanto al desarrollo científico interdisciplinario, ya que aísla a los investigadores en sus respectivas temáticas sin posibilidades de interacción con los de otros campos disciplinares.

Como corolario de lo expuesto se desarrollan subculturas con un perfil muy consistente en las diversas Facultades, que dificultan los acuerdos y aún el simple entendimiento entre docentes universitarios provenientes de diferentes orígenes.

Como segunda cuestión, y no menos importante que la anterior, surge la falta de aplicación del conocimiento específico a su propia gestión. Las universidades enseñan a gestionar todo tipo de organizaciones, desarrollando carreras de grado y postgrado al respecto, pero ellas mismas rara vez aplican los principios y técnicas que enseñan, a su propia gestión.

Debemos asumir que, en la medida que nuestras universidades sigan siendo autogestionadas por sus miembros y es de desear que esto continúe, estamos siendo gerenciados por "aficionados", ya que no otra cosa resulta cualquier graduado universitario al que la fortuna y el voto de la comunidad lo llevan a ejercer puestos de conducción (Rectores, Decanos, Consejeros, etc.).

Es de destacar que frente a esto se observa actualmente una incipiente reacción con la aparición de Maestrías y Tecnicaturas orientadas a la gestión de la educación superior, así como se suceden Congresos y Coloquios dedicados a esa problemática y aún se verifica un intento por lograr algún nivel de coordinación en el desarrollo de dichos cursos ${ }^{7}$.

\footnotetext{
${ }^{7}$ Los encuentros más notorios son los que llevan por título "La universidad como objeto de investigación" y "Coloquios de Gestión Universitaria de América del Sur", que llevan varios eventos realizados. Se ha creado asimismo una red de Postgrados que tienen a la Universidad como objeto (Rapes), que realiza reuniones periódicas de los responsables de los mismos.
}

Rev. GUAL., Florianópolis, v.2, n. 1, p.47-61, ano 2009. 


\section{LÍNEAS DE ACCIÓN A DESARROLLAR}

\subsection{En General}

$\underline{\text { Necesidad de situarse en la condición de País Subdesarrollado }}$

Nuestro país viene sobrellevando desde hace varias décadas una crisis del llamado “Estado de Bienestar" que remató en la década del 90' con la aplicación a rajatabla de las doctrinas neoliberales del llamado "consenso de Washington", que terminó con la desarticulación de su aparato productivo y la enajenación de sus sectores de servicios estratégicos y aún los de energía, con su secuela de endeudamiento, empobrecimiento y desocupación, que concluyó dramáticamente con la crisis del año 2001

Frente a esto nuestro país viene reaccionando empíricamente, con marchas y contramarchas, pero sin un sustento teórico que permita visualizar claramente políticas de mediano y largo plazo.

Por lo expuesto y frente a la crisis del "socialismo real" y a la falacia que implica abrazar doctrinas neoliberales que han demostrado palmariamente su inconsistencia para garantizar nuestro desarrollo, se hace imperioso plantear un esfuerzo sistemático de nuestras ciencias sociales para elaborar un modelo teórico alternativo que guíe el desarrollo de políticas de Estado consistentes.

\section{Desde esta perspectiva la Universidad debe:}

- Desarrollar una cultura que reconozca y genere conductas basadas En:

-La necesidad del uso de conocimiento intensivo en todos los ámbitos de la Universidad, no sólo en sus aulas, laboratorios y gabinetes de investigación. Al respecto es importante que se entienda que cuanto más alto en la pirámide jerárquica puedan aplicarse los saberes pertinentes, mayor será el beneficio que la Universidad perciba. A contrario sensu cuanto más sea la ignorancia en estos niveles, mayor será el perjuicio global que se ocasione.

- Que el progreso está atado a la innovación. No por ser ésta una verdad de perogrullo resulta menos importante destacarlo en este momento, ya que la Universidad como organización resulta ser la más refractaria a los cambios en su interior, aún cuando los impulsa en los campos científicos que aborda. Para corroborar lo que aquí se afirma basta observar las ofertas de carreras, las estructuras organizativas y académicas y la cultura organizacional de nuestras universidades para ver cuan poco han cambiado en los últimos cincuenta años. Y lo que es aún peor, se 
verifica que muchas de las nuevas universidades surgidas en dicho lapso nacieron "viejas" o sea resultaron copias de otras ya instaladas.

- La obsolescencia de los saberes impone la revisión permanente de las soluciones adoptadas. Cualquiera que circule por nuestras universidades y sin ser especialista en Análisis Organizacional intente averiguar por que se hacen determinadas cosas de un modo u otro, recibirá en la mayoría de los casos la respuesta "porque siempre se hicieron así". Aquí se verifica una vez más que lo que la Universidad enseña acerca de la obsolescencia de los conocimientos, no tiene aplicación en su ámbito interno.

- Que los problemas son complejos y reconocen multicausalidad, por lo que las soluciones deben buscarse en forma interdisciplinaria. Los especialistas en organizaciones afirman que la Universidad es, en si misma, una de las organizaciones más complejas que se conocen. Contribuye a ello la diversidad de grupos humanos que la habitan, ya sea por su rol (docentes, funcionariado, alumnos, investigadores, etc.), las subulturas que genera, la difusión del poder que conlleva su sistema de gobierno, la multiplicidad de fines que persigue, los intereses y expectativas de sectores externos a la misma que intentan presionar sobre ella, entre otras causales. Frente esto la tendencia, tal como se ha señalado, es al aislacionismo y esto constituye un rasgo cultural que debe ser erradicado.

- Reconocer en la Universidad la existencia de:

- Un Capital Humano que posee actitudes (emotivas) y aptitudes (saberes) que es preciso preserva y enriquecer. Es notorio el compromiso que su claustro docente ha demostrado a través de la historia de nuestra Universidad, sobrellevando condiciones laborales deficitarias, con salarios magros y haciéndose cargo de tareas de conducción que insumen horas y no tienen más retribución que la satisfacción de reconocerse elegidos por sus pares. Asimismo resulta notorio que, salvo excepciones, son ellos mismos quienes deben sobrellevar el costo de su propio perfeccionamiento, aún cuando lo hacen en cuestiones que tienen por destinataria a la propia Universidad (tal el caso de los postgrados en docencia o gestión universitarias).

Otro tanto puede afirmarse del personal de apoyo de nuestras universidades, que salvo excepciones, no recibe una capacitación consistente ni un reconocimiento a sus esfuerzos personales por hacerlo.

- Un Capital Estructural que se basa en la calidad organizativa y tecnológica de la Universidad, pero que debe ser atendido para evitar la entropía propia de todo sistema y su degradación inexorable. Esto implica que la propia Universidad se dote 
de la tecnoestructura ${ }^{8}$ necesaria para atender esta cuestión y mantener vigente su calidad.

- Un Capital Social que determina calidad de la vida social, solidaridad, respeto a las normas e instituciones y que genera un determinado clima organizacional. Debemos reconocer a la luz de los episodios que vienen dándose en el seno de nuestras universidades y que en algunos casos han llegado a impedir la elección de sus autoridades y las reformas de sus estatutos, que este capital está muy devaluado entre nosotros y es preciso una labor muy seria y perseverante para recrearlo.

\subsection{En Particular:}

En este punto nos detendremos en las acciones que entendemos deben darse en los distintos niveles y estamentos de la Universidad:

\section{$\underline{\text { A nivel Conducción }}$}

En este nivel es preciso señalar que nuestras universidades adolecen de una terrible confusión de roles decisorios ${ }^{9}$, lo que genera que órganos superiores, que debieran realizar la conducción política de la Universidad, estén resolviendo cuestiones meramente operativas y a la inversa, otros de nivel intermedio (Consejos Directivos o Académicos) sean los que proponen las líneas a seguir (creación de carreras, direccionamiento de la investigación, etc.). Frente a esto es preciso comprender que en la Universidad, como en cualquier organización, existen tres niveles diferenciados que deben cumplir cada uno de ellos sus respectivos roles, sin interferir en los restantes. Estos niveles son:

- Nivel Político. Representado por el órgano máximo (Asamblea), es quien debe fijar los lineamientos políticos y los valores a sustentar. Esto implica definir la Estructura de la Universidad, su territorio y población de interés, sus áreas de conocimiento y problemáticas sociales prioritarias.

- Nivel de Gobierno (Consejos Superiores) deben tener a su cargo decisiones necesarias para implementar las políticas fijadas, desarrollando la acción normativa necesaria, planificando y asignando recursos y efectuando el control de gestión necesario. En este punto es necesario señalar que nuestra tradición ha hecho que el cuerpo encargado de este nivel decisorio, asuma también el rol de "Órgano Jurisdiccional" o árbitro final de todo reclamo o controversia que se suscite en el

\footnotetext{
${ }^{8}$ Concepto creado por H. Mintzberg -1988- que identifica un sector de la organización especialmente dedicado al análisis de su estructura, sus procedimientos, las capacidades necesarias para su gestión y los escenarios potenciales a los que la organización deberá enfrentarse.

${ }^{9}$ Este problema ha sido extensamente tratado en mi artículo publicado en "Faces" n* 2, año 2, 1996 , pag. 96 y sigts. Revista de la Facultad de Cs. Económicas y Sociales de la UNMDP.
} 
ámbito universitario. De hecho esta tarea ocupa mayoritariamente el tiempo de trabajo de este cuerpo y sería altamente aconsejable crear otro ente especializado (podría llamarse Senado Académico), para ocuparse exclusivamente de esta función.

- Nivel de Gerencia. Implica las decisiones técnicas necesarias para implementar los planes y programas y aplicar los recursos. En nuestro caso se trata de cargos unipersonales (Rector y Decanos) y eventualmente los Consejos Directivos o Académicos. Al respecto es importante señalar aquí que es precisamente la falta de aplicación de técnicas de planificación y control de gestión, que por otra parte la Universidad desarrolla y enseña a sus alumnos, la que determina que órganos colegiados con funciones de Gobierno, estén permanentemente involucrados en actividades de Gerencia, al controlar acción por acción la gestión de los responsables de la misma.

\section{$\underline{\text { A Nivel Docencia }}$}

Los docentes son el recurso básico de toda Universidad y de su calidad dependerá a no dudarlo, gran parte del éxito de la misma. No pretendemos aquí minimizar la importancia de otros actores y factores, pero no caben dudas que la excelencia de este sector es, sino un requisito suficiente, si un requisito necesario.

Respecto del mismo entonces es preciso tener políticas realistas y con recursos asignados para permitir su capacitación permanente. Resulta lamentable constatar que aún en el concierto de la Universidad Latinoamericana nuestra Universidad está seriamente rezagada en cuanto a docentes con títulos de posgraduación y doctorados.

Al respecto es de destacar una medida reciente de la Secretaría de Políticas Universitarias que estableció un plus salarial para los docentes que hayan alcanzado un doctorado. Sería de desear que en un par de décadas ningún docente que alcance la titularidad carezca de dicho título.

Paralelamente a esto debería revisarse el modelo de asignación docente "por cátedra" que conlleva la estructuración de pirámides aisladas, en las que sólo la muerte, deserción o jubilación de su cabeza (titular) abre espacio para quienes lo secundan. Esto lleva también al desaliento y la frustración cuando se observa, en algunas disciplinas, docentes doctorados que fungen como auxiliares de docencia. Bastaría modificar el criterio y designar los profesores por área de conocimiento y dentro de ella asignar categorías no en función de cátedras sino de méritos.

En cualquier caso es necesario tener presente que este cuerpo debe capacitarse como mínimo en cuatro campos: 
- Formación Científica. Este campo es el que se encuentra actualmente más estructurado, con el surgimiento de gran cantidad de postgrados (especializaciones, maestrías y doctorados) que permiten a quienes están motivados, progresar en su formación y hacerlo en forma gradual y permanente. Lamentablemente este crecimiento ha sido también anárquico y por lo mismo no cubre homogéneamente todas las disciplinas ni todo el territorio de la Nación. Por otra parte no tiene, en general, una financiación ad hoc, por lo que los mismos interesados deben costear su desarrollo so pena de que los cursos se cierren.

- Formación para la enseñanza. Esta formación, mal llamada pedagógica ya que este término se aplica a la enseñanza a niños, resulta imprescindible para los docentes universitarios, cuya inmensa mayoría posee una razonable formación científica, pero carece absolutamente de las herramientas mínimas para hacer accesible dichos conocimientos a sus alumnos.

Al respecto debemos puntualizar que aún es necesaria mucha investigación aplicada en el campo de las Ciencias de la Educación para desarrollar metodologías aptas para la Universidad y para las diversas áreas de conocimiento que en la misma se encuentran.

- Formación para la Gestión. Venimos sosteniendo en este trabajo que la Universidad es una organización compleja y de la calidad de su gestión dependerá, como en cualquier otra organización, la mayor posibilidad de éxito o fracaso. Aquí es posible afirmar que si bien una buena Gestión no convierte a una Universidad con docentes mediocres en una gran Universidad, una mala gestión puede anular el esfuerzo del mejor cuerpo docente.

Ya consignamos anteriormente que se observa una toma de conciencia al respecto y que han surgido algunos postgrados y diversos foros donde este tema ha sido considerado. No obstante es necesaria una política manifiesta que reconozca la necesidad de que, al menos quienes tengan vocación de liderazgo, transiten por algún nivel de preparación en esta temática. Paralelamente sería deseable que si no en todas las Universidades, sí en todas las regiones, mediante acuerdos interuniversitarios, se organicen postgrados que tengan a la propia Universidad como objeto de estudio.

- Formación para la Interdisciplina. Venimos sosteniendo que las problemáticas del medio socioproductivo son esencialmente multisdisciplinares, por lo tanto resulta obvio que quienes deban encarar su análisis y desarrollo posean mínimas actitudes y aptitudes para el trabajo interdisciplinario.

Nuestra propia experiencia en las tareas de Gestión Universitaria nos ha permitido observar las dificultades que es preciso salvar para trabajar cualquier problema con docentes 
provenientes de distintas áreas disciplinares. Hay diferencias de percepción, de lenguaje, de enfoque que dificultan un diálogo fructífero. No obstante esta tarea es necesaria y la Universidad debe hacer un esfuerzo por lograrlo.

Algunas han iniciado este camino creando Institutos de Investigación y Desarrollo Interdisciplinarios (Cuyo es un ejemplo que conozco) abocados a determinadas problemáticas del contexto. Pensamos que es un camino digno de imitarse.

\section{$>$ A Nivel Funcionariado de Apoyo}

Toda organización debe contar para su correcto funcionamiento, con personal calificado para las tareas a realizar, y la Universidad no es una excepción. Muy por el contrario su particular configuración, en la cual necesariamente coexisten un grupo dedicado realizar lo que podríamos llamar las "actividades fines" (docentes) y el otro dedicado a todas las actividades de apoyo que son necesarias para que aquellas antes citadas puedan llevarse a cabo (funcionariado o no docentes), hace indispensable pensar en que ambos, acorde a su particular rol en la organización, deben poseer la mayor idoneidad posible.

Pero esta situación se ve agravada en la Universidad Argentina por su calidad de Ente Autogestionado, en el cual los docentes asumen roles de Gobierno y Gerencia que tienen por característica su periodicidad, donde necesitan en todo momento del asesoramiento y asistencia del personal permanente - funcionariado- que por lo que ya consignáramos antes respecto a la falta de preparación para el desempeño de dichos roles, dependen en grado sumo de la capacidad, responsabilidad y sentido ético de estos últimos.

Acorde a lo expuesto se hace evidente la necesidad de proceder con el mayor celo en el reclutamiento de este personal, pero también surge la demanda de capacitación de los mismos para permitir su desarrollo futuro y el cubrimiento de las diversas funciones que la Universidad demanda.

Atento a esto debe tenerse presente que dentro de este grupo es posible efectuar una primera gran división:

- Personal de Administración. Que incluye a todos los que realizan tareas vinculadas la registración, circulación, e interpretación de la información que fluye en los diferentes procesos administrativos, así como implica la colaboración en la toma de decisiones de las autoridades respectivas, aportando información de base $y$ conocimiento técnico. 
Este personal, más allá de las calificaciones profesionales que algunos puestos demanden, requiere una capacitación que le permita entender la complejidad de los procesos que se desenvuelven en la Universidad, así como las diferentes técnicas de manejo de la información que son necesarias para su correcto manejo.

Sin duda lo anterior exige contar, como ya lo señaláramos, de una Tecnoestructura que pueda discernir las competencias necesarias para los diferentes cargos, pero también identificar las carencias que evidencian quienes los desempeñan, para de ese modo poder encarar acciones remediales de capacitación.

- Personal Técnico. Involucramos aquí una variada gama de actividades que van desde servicios de informática, mantenimiento general, gestión de laboratorios y talleres, servicios de salud, y toda una variada gama de técnicas que dependerán, en todo caso, de la particular conformación de cada Universidad.

Respecto de este personal se hace evidente que, en la mayoría de los casos, se requerirá de los mismos una calificación previa, pero esto no elimina la necesidad de continuar esta capacitación para ajustarla a las particulares demandas de las tareas a desempeñar. Cabe aquí la misma reflexión que se formula en el último párrafo del punto anterior.

Para cerrar este punto podemos apuntar que, cualquiera sea el tipo de personal de apoyo al que hagamos referencia, siempre será útil pensar en desarrollar Tecnicaturas en Gestión Universitaria, con las orientaciones adecuadas, que permitirán al personal no sólo capacitarse, sino avanzar en su desarrollo personal.

No es lícito pensar que la organización que tiene el conocimiento como materia prima de su tarea, no haga nada para que el mismo llegue a todos quienes están en contacto con ella, posibilitando a un mismo tiempo su desarrollo organizacional y el de las personas que con ella colaboran.

\section{CONCLUSIONES}

Para cerrar este pequeño ensayo nos parece útil dejar plasmadas algunas ideas fuerza, que apunten al logro de lo planteado en la Introducción de este trabajo. 
1- Planificar y facilitar la capacitación y el desarrollo de los miembros de la Universidad. Esta tarea podrá darse mediante becas, pasantías, cursos de grado y postgrado, Tecnicaturas, cursos remediales y cualquier otro medio que resulte idóneo.

2- $\quad$ Premiar la capacitación y el desarrollo personal. Esto implica plantear la selección y calificación del personal a todo nivel (tanto docente como funcionariado) y establecer mecanismos de retribución, jerarquización, otorgamiento de distinciones, y cualquier otro mecanismo gratificatorio que se elabore, que tengan en cuenta los esfuerzos de cada uno en pos de su mejoramiento y calificación.

3- Ubicar los saberes donde deben emplearse. Debemos tener presente que la mera antigüedad no garantiza el conocimiento y que, además, éste obsolece, por lo que se deberá estar atento a que en cada cargo (tanto docente, como de gestión o apoyo) se ubique la persona con mayor idoneidad para el mismo.

4- $\quad$ Sinergizar los saberes mediante la labor interdisciplinaria. Insistimos en este punto por la fuerte tendencia que se registra en nuestras universidades al aislacionismo y a analizar cualquier situación a la luz de la propia y restringida visión.

Por último y a modo de cierre vayan estas tres frases que resumen nuestro pensamiento:

\section{* FORMACIÓN ETICA Y COMPROMISO SOCIAL; \\ * DESALENTAR LA MEDIOCRIDAD; \\ * GeSTIONAR EL CONOCIMIENTO DESDE EL CONOCIMIENTO.}

\section{BIBLIOGRAFÍA BÁSICA CONSULTADA}

ARANA, Marta "El Programa de Incentivos bajo la lupa: El caso UNMDP”; 2005. Edit. FRONDIZI, Risieri. "La Universidad en un mundo de tensiones".1971. Edit. Paidos.

GARCIA de FANELLI, Ana María. Universidad, Organización e inventivos”. 2005. Edit. Miño y Dávila.

MONCADA, Alberto. “Administración Universitaria”. 1971 Editor Fundación Moncada Kayon. Madrid.

MINTZBERG, Henry. "La estructuración de las organizaciones". 1984 Editorial Ariel Barcelona. 
PARRINO, María del Carmen. "Capital Académico. Una mirada sobre la gestión del cuerpo docente universitario" 2007. AV Ediciones para profesionales.

SCHUMPETER, Joseph. "Teoría del desenvolvimiento económico" 1997 última edición. Fondo de Cultura Económica. México.

SUTZ, Judith. "Universidad Y Sectores Productivos”. 1994. Centro Editor de América Latina.

TENTI FANFANI, Emilio (Compilador) Universidad y Empresa"1993. Editorial Miño y Dávila.

TROTTINI Ana María. "La especificidad de la Gestión Universitaria: Gobierno y administración de su capital humano". 2007. Editor U. Nacional de Rosario.

VEGA, Roberto I. “La Universidad Argentina: una institución en crisis?”.1996, revista de la Facultad de Ciencias Económicas y Sociales de la UNMDP "Faces", Año 2, n*2, pag. 94 y sgts. 